\title{
The angular spin current and its physical consequences
}

\author{
Qing-feng $\operatorname{Sun}^{1, *}$ and X. C. Xie ${ }^{2,3}$ \\ ${ }^{1}$ Beijing National Lab for Condensed Matter Physics and Institute of Physics, \\ Chinese Academy of Sciences, Beijing 100080, China \\ ${ }^{2}$ Department of Physics, Oklahoma State University, Stillwater, Oklahoma 74078 \\ ${ }^{3}$ International Center for Quantum structures, Chinese Academy of Sciences, Beijing 100080, China
}

(Dated: September 5, 2018)

\begin{abstract}
We find that in order to completely describe the spin transport, apart from spin current (or linear spin current), one has to introduce the angular spin current. The two spin currents respectively describe the translational and rotational motion of a spin. The definitions of these spin current densities are given and their physical properties are discussed. Both spin current densities appear naturally in the spin continuity equation. Moreover we predict that the angular spin current can also induce an electric field $\vec{E}$, and in particular $\vec{E}$ scales as $1 / r^{2}$ at large distance $r$, whereas the $\vec{E}$ field generated from the linear spin current goes as $1 / r^{3}$.
\end{abstract}

PACS numbers: 72.25.-b, 85.75.-d, 73.23.-b

Recently, a new sub-discipline of condensed matter physics, spintronics, is emerging rapidly and generating great interests. 1, 2] The spin current, the most important physical quantity in spintronics, has been extensively studied. Many interesting and fundamental phenomena, such as the spin Hall effect [3, 4, 5] and the spin precession $[\underline{6}$, 7] in systems with spin-orbit coupling, have been discovered and are under further study.

As for the charge current, the definition of the local charge current density $\vec{j} e(\mathbf{r}, t)=\operatorname{Re}\left[\Psi^{\dagger}(\mathbf{r}, t) \vec{v} \Psi(\mathbf{r}, t)\right]$ and its continuity equation $\frac{d}{d t} \rho(\mathbf{r}, t)+\nabla \bullet \vec{j} e(\mathbf{r}, t)=0$ is well-known in physics. Here $\Psi(\mathbf{r}, t)$ is the electronic wave function, $\vec{v}=\dot{\mathbf{r}}$ is the velocity operator, and $\rho(\mathbf{r}, t)=\Psi^{\dagger} \Psi$ is the charge density. This continuity equation is the consequence of charge invariance, i.e. when an electron moves from one place to another, its charge remains the same. However, in the spin transport, there are still a lot of debates over what is the correct definition for spin current. The problem stems from the lack of understanding of the spin continuity equation. Recently, some studies have begun investigation in this direction, e.g. a semi-classical description of the spin continuity equation has been proposed 8 , 9].

In this paper, we study the quantum version of the continuity equation and the definition of local spin current density. We find that in order to completely describe the spin transport, one has to use two quantities, the linear spin current and the angular spin current, which respectively describe the translational and rotational motion of a spin. The conventional linear spin current has been extensively studied. However, the angular spin current has not been investigated before. The definition of two spin current densities are given and they appear naturally in the quantum spin continuity equation. Moreover, we predict that the angular spin current can generate an electric field similar as with the linear spin current, and thus contains physical consequences.

Before studying the spin current in a quantum system, we first consider the classical case. Consider a classical particle having a vector $\vec{m}$ (e.g. the classical magnetic moment, etc.) with its magnitude $|\vec{m}|$ fixed under the particle motion. To completely describe this vector flow (see Fig.1a), we need three quantities: the local vector density $\vec{M}(\mathbf{r}, t)=\rho(\mathbf{r}, t) \vec{m}(\mathbf{r}, t)$, the velocity $\vec{v}(\mathbf{r}, t)$, and the angular velocity $\vec{\omega}(\mathbf{r}, t)$. Here $\rho(\mathbf{r}, t)$ is the particle density, and $\vec{v}$ and $\vec{\omega}$ describe the translational and rotational motion, respectively. Since $|\vec{m}|$ is a constant, the continuity equation is (see Fig.1a): $\frac{d}{d t} \iiint_{V} \vec{M} d V=-\oint_{S} d \vec{S} \bullet \vec{v} \vec{M}+\iiint_{V} \vec{\omega} \times \vec{M} d V$, or it can be rewritten in the differential form:

$$
\frac{d}{d t} \vec{M}(\mathbf{r}, t)=-\nabla \bullet \vec{v}(\mathbf{r}, t) \vec{M}(\mathbf{r}, t)+\vec{\omega}(\mathbf{r}, t) \times \vec{M}(\mathbf{r}, t),
$$

where $\vec{v} \vec{M}$ is a tensor, and its element $(\vec{v} \vec{M})_{i j}=v_{i} M_{j}$. 
Now we study the electronic spin $\vec{s}$ in the quantum case. Consider an arbitrary wave function $\Psi(\mathbf{r}, t)$. The local spin density $\vec{s}$ at the position $\mathbf{r}$ and time $t$ is: $\vec{s}(\mathbf{r}, t)=\Psi^{\dagger}(\mathbf{r}, t) \hat{\vec{s}} \Psi(\mathbf{r}, t)$, where $\hat{\vec{s}}=\frac{\hbar}{2} \hat{\vec{\sigma}}$ with $\hat{\vec{\sigma}}$ being the Pauli matrices. The time-derivative of $\vec{s}(\mathbf{r}, t)$ is:

$$
\frac{d}{d t} \vec{s}(\mathbf{r}, t)=\frac{\hbar}{2}\left\{\left[\frac{d}{d t} \Psi^{\dagger}\right] \hat{\vec{\sigma}} \Psi+\Psi^{\dagger} \hat{\vec{\sigma}} \frac{d}{d t} \Psi\right\} .
$$

From the Schrodinger equation, we have $\frac{d}{d t} \Psi(\mathbf{r}, t)=\frac{1}{i \hbar} H \Psi(\mathbf{r}, t)$ and $\frac{d}{d t} \Psi^{\dagger}(\mathbf{r}, t)=\frac{1}{-i \hbar}[H \Psi(\mathbf{r}, t)]^{\dagger}$. Notice here the transposition in the symbol $\dagger$ only acts on the spin indexes. By using the above two equations, the Eq.(2) changes into:

$$
(d / d t) \vec{s}(\mathbf{r}, t)=\left[\Psi^{\dagger} \hat{\vec{\sigma}} H \Psi-(H \Psi)^{\dagger} \hat{\vec{\sigma}} \Psi\right] / 2 i
$$

In the derivation below, we use the following Hamiltonian:

$$
H=\frac{\vec{p}^{2}}{2 m}+V(\mathbf{r})+\hat{\vec{\sigma}} \bullet \vec{B}+\frac{\alpha}{\hbar} \hat{z} \bullet(\hat{\vec{\sigma}} \times \vec{p}) .
$$

Note that our results are independent of this specific choice of the Hamiltonian. 10] In Eq.(4) the 1st and 2nd terms are the kinetic energy and potential energy. The 3rd term is the Zeeman energy due to a magnetic field, and the last term is the Rashba spin-orbit coupling [11, 12], which has been extensively studied recently [4, 6, 7]. Next we substitute the Hamiltonian Eq.(4) into Eq.(3), and calculate the right side of Eq.(3) one term by one term:

(1). For the 1 st term of Eq.(4), $\frac{\vec{p}^{2}}{2 m}$ :

$$
\begin{aligned}
\Psi^{\dagger} \hat{\vec{\sigma}} & \left.\vec{p}^{2} / 2 m\right) \Psi-\left[\left(\vec{p}^{2} / 2 m\right) \Psi\right]^{\dagger} \hat{\vec{\sigma}} \Psi \\
& =-\left(\hbar^{2} / 2 m\right)\left\{\Psi^{\dagger} \hat{\vec{\sigma}} \nabla \bullet \nabla \Psi-[\nabla \bullet \nabla \Psi]^{\dagger} \hat{\vec{\sigma}} \Psi\right\} \\
& =-\left(\hbar^{2} / 2 m\right) \nabla \bullet\left\{\Psi^{\dagger} \nabla \hat{\vec{\sigma}} \Psi-[\nabla \Psi]^{\dagger} \hat{\vec{\sigma}} \Psi\right\} \\
& =-i \hbar \nabla \bullet \operatorname{Re}\left\{\Psi^{\dagger}(\vec{p} / m) \hat{\vec{\sigma}} \Psi\right\}
\end{aligned}
$$

Here the dotted multiply between the vector and the tensor is defined as usually, e.g. $(\vec{A} \bullet \mathbf{B})_{j} \equiv \sum_{i} A_{i} B_{i j}$ with the vector $\vec{A}$ and the tensor $\mathbf{B}$.

(2). For the 2 nd term of Eq.(4), $V(\mathbf{r})$ : its corresponding value is zero.

(3). For the 3 rd term, $\hat{\vec{\sigma}} \bullet \vec{B}$ :

$$
\begin{aligned}
& \Psi^{\dagger} \hat{\vec{\sigma}}(\hat{\vec{\sigma}} \bullet \vec{B}) \Psi-[\hat{\vec{\sigma}} \bullet \vec{B} \Psi]^{\dagger} \hat{\vec{\sigma}} \Psi \\
& \quad=\Psi^{\dagger}(\hat{\vec{\sigma}} \hat{\vec{\sigma}} \bullet \vec{B}-\vec{B} \bullet \hat{\vec{\sigma}} \hat{\vec{\sigma}}) \Psi=2 i \Psi^{\dagger} \vec{B} \times \hat{\vec{\sigma}} \Psi
\end{aligned}
$$

(4). For the last term, $\frac{\alpha}{\hbar} \hat{z} \bullet(\hat{\vec{\sigma}} \times \vec{p})=\frac{\alpha}{\hbar} \hat{\vec{\sigma}} \bullet(\vec{p} \times \hat{z})$ :

$$
\begin{aligned}
& \Psi^{\dagger} \hat{\vec{\sigma}} \hat{\vec{\sigma}} \bullet(\vec{p} \times \hat{z}) \Psi-[\hat{\vec{\sigma}} \bullet(\vec{p} \times \hat{z}) \Psi]^{\dagger} \hat{\vec{\sigma}} \Psi \\
& =\Psi^{\dagger}(\vec{p} \times \hat{z}) \bullet \hat{\vec{\sigma}} \hat{\vec{\sigma}} \Psi+\Psi^{\dagger} 2 i(\vec{p} \times \hat{z}) \times \hat{\vec{\sigma}} \Psi-[\hat{\vec{\sigma}} \bullet(\vec{p} \times \hat{z}) \Psi]^{\dagger} \hat{\vec{\sigma}} \Psi \\
& =-i \hbar\left\{\Psi^{\dagger}\left(\nabla \bullet(\hat{z} \times \hat{\vec{\sigma}}) \hat{\vec{\sigma}} \Psi+[\nabla \Psi]^{\dagger} \bullet(\hat{z} \times \hat{\vec{\sigma}}) \hat{\vec{\sigma}} \Psi\right\}+2 i \Psi^{\dagger}(\vec{p} \times \hat{z}) \times \hat{\vec{\sigma}} \Psi\right. \\
& =-i \hbar \nabla \bullet\left\{\Psi^{\dagger}(\hat{z} \times \hat{\vec{\sigma}}) \hat{\vec{\sigma}} \Psi\right\}+2 i \Psi^{\dagger}(\vec{p} \times \hat{z}) \times \hat{\vec{\sigma}} \Psi
\end{aligned}
$$

In the similar method, one also has:

$$
\begin{aligned}
& \Psi^{\dagger} \hat{\vec{\sigma}} \hat{\vec{\sigma}} \bullet(\vec{p} \times \hat{z}) \Psi-[\hat{\vec{\sigma}} \bullet(\vec{p} \times \hat{z}) \Psi]^{\dagger} \hat{\vec{\sigma}} \Psi \\
& \quad=-i \hbar \nabla \bullet\left\{\Psi^{\dagger}(\hat{z} \times \hat{\vec{\sigma}}) \hat{\vec{\sigma}} \Psi\right\}^{*}+2 i[(\vec{p} \times \hat{z}) \times \hat{\vec{\sigma}} \Psi]^{\dagger} \Psi
\end{aligned}
$$


Therefore, we obtain the result of the last term:

$$
\begin{aligned}
& (\alpha / \hbar)\left\{\Psi^{\dagger} \hat{\vec{\sigma}} \hat{\vec{\sigma}} \bullet(\vec{p} \times \hat{z}) \Psi-[\hat{\vec{\sigma}} \bullet(\vec{p} \times \hat{z}) \Psi]^{\dagger} \hat{\vec{\sigma}} \Psi\right\} \\
& =-i \alpha \nabla \bullet \operatorname{Re} \Psi^{\dagger}(\hat{z} \times \hat{\vec{\sigma}}) \hat{\vec{\sigma}} \Psi+\frac{2 i \alpha}{\hbar} \operatorname{Re} \Psi^{\dagger}(\vec{p} \times \hat{z}) \times \hat{\vec{\sigma}} \Psi
\end{aligned}
$$

To summarize the above four terms, the Eq.(3) changes into:

$$
\begin{aligned}
\frac{d}{d t} \vec{s} & =-\frac{\hbar}{2} \nabla \bullet \operatorname{Re}\left\{\Psi^{\dagger}\left[\frac{\vec{p}}{m}+\frac{\alpha}{\hbar}(\hat{z} \times \hat{\vec{\sigma}})\right] \hat{\vec{\sigma}} \Psi\right\} \\
& +\operatorname{Re}\left\{\Psi^{\dagger}\left[\vec{B}+\frac{\alpha}{\hbar} \vec{p} \times \hat{z}\right] \times \hat{\vec{\sigma}} \Psi\right\} .
\end{aligned}
$$

Introducing a tensor $\mathbf{j}_{s}(\mathbf{r}, t)$ and a vector $\vec{j}_{\omega}(\mathbf{r}, t)$ :

$$
\begin{aligned}
& \mathbf{j}_{s}(\mathbf{r}, t)=\operatorname{Re}\left\{\Psi^{\dagger}\left[\frac{\vec{p}}{m}+\frac{\alpha}{\hbar}(\hat{z} \times \hat{\vec{\sigma}})\right] \hat{\vec{s}} \Psi\right\} \\
& \vec{j}_{\omega}(\mathbf{r}, t)=\operatorname{Re}\left\{\Psi^{\dagger} \frac{2}{\hbar}\left[\vec{B}+\frac{\alpha}{\hbar}(\vec{p} \times \hat{z})\right] \times \hat{\vec{s}} \Psi\right\}
\end{aligned}
$$

then Eq.(3) reduces to:

$$
\frac{d}{d t} \vec{s}(\mathbf{r}, t)=-\nabla \bullet \mathbf{j}_{s}(\mathbf{r}, t)+\vec{j}_{\omega}(\mathbf{r}, t),
$$

or it can also be rewritten in the integral form:

$$
\frac{d}{d t} \iiint_{V} \vec{s} d V=-\oint_{S} d \vec{S} \bullet \mathbf{j}_{s}+\iiint_{V} \vec{j}_{\omega} d V .
$$

Due to the fact that $\hat{\vec{v}}=\frac{d}{d t} \mathbf{r}=\frac{\vec{p}}{m}+\frac{\alpha}{\hbar}(\hat{z} \times \hat{\vec{\sigma}})$ and $\frac{d}{d t} \hat{\vec{\sigma}}=\frac{1}{i \hbar}[\hat{\vec{\sigma}}, H]=\frac{2}{\hbar}\left[\vec{B}+\frac{\alpha}{\hbar} \vec{p} \times \hat{z}\right] \times \hat{\vec{\sigma}}$, Eqs. $(9)$ and (10) become:

$$
\begin{aligned}
& \mathbf{j}_{s}(\mathbf{r}, t)=\operatorname{Re}\left\{\Psi^{\dagger}(\mathbf{r}, t) \hat{\vec{v}} \hat{\vec{s}} \Psi(\mathbf{r}, t)\right\} \\
& \vec{j}_{\omega}(\mathbf{r}, t)=\operatorname{Re}\left\{\Psi^{\dagger}(d \hat{\vec{s}} / d t) \Psi\right\}=\operatorname{Re}\left\{\Psi^{\dagger} \hat{\vec{\omega}} \times \hat{\vec{s}} \Psi\right\}
\end{aligned}
$$

where $\hat{\vec{\omega}} \equiv \frac{2}{\hbar}\left[\vec{B}+\frac{\alpha}{\hbar}(\vec{p} \times \hat{z})\right] \cdot[10]$

Eq.(11) is the spin continuity equation, which is very similar with the classic vector continuity equation (1). In fact, this spin continuity equation (11) is the consequence of invariance of the spin magnitude $|\vec{s}|$, i.e. when an electron makes a motion, either translation or rotation, its spin magnitude $|\vec{s}|=\frac{\hbar}{2}$ remains a constant. The two quantities $\mathbf{j}_{s}(\mathbf{r}, t)$ and $\vec{j}_{\omega}(\mathbf{r}, t)$ in Eq. $(11)$, which are defined in Eqs. $(13,14)$ respectively, describe the translational and rotational motion of a spin at the location $\mathbf{r}$ and the time $t$. They will be named the linear and the angular spin current densities accordingly $13 \mid$. In fact, the linear spin current $\mathbf{j}_{s}(\mathbf{r}, t)$ is identical with the conventional spin current investigated in recent studies [14]. However, the angular spin current $\vec{j}_{\omega}(\mathbf{r}, t)$ is new and has not been investigated before. 13]

Next, we discuss certain properties of $\mathbf{j}_{s}(\mathbf{r}, t)$ and $\vec{j}_{\omega}(\mathbf{r}, t)$. Notice that $\vec{j}_{\omega}(\mathbf{r}, t)$ which describe the rotational motion of the spin plays a parallel role in comparison with the conventional linear spin current $\mathbf{j}_{s}(\mathbf{r}, t)$ for the spin transport. (1) The linear spin current is a tensor. Its element, e.g. $j_{s, x y}$, represents an electron moving along the $x$ direction with its spin in the $y$ direction (see Fig.1b). The angular spin current $\vec{j}_{\omega}$ is a vector. In Fig.1c, its element $j_{\omega, x}$ describes the rotational motion of the spin in the $y$ direction and the angular velocity $\vec{\omega}$ in the $-z$ direction. (2) Why is it necessary to introduce two quantities instead of one to describe a spin motion? The reason is the same as for completely describing the motion of a classic vector or a rigid body. There two quantities are used: the linear velocity for the translational motion and the angular velocity for the rotation. It is similar for the present quantum case, both 
$\mathbf{j}_{s}(\mathbf{r}, t)$ and $\vec{j}_{\omega}(\mathbf{r}, t)$ are required to describe the motion of a quantum spin. (3) From the linear spin current density $\mathbf{j}_{s}(\mathbf{r}, t)$, one can calculate (or say how much) the linear spin current $\vec{I}_{s}$ flowing through a surface $S$ (see Fig.1e): $\vec{I}_{s}^{S}=\iint_{S} d \vec{S} \bullet \mathbf{j}_{s}$. However, the behavior for the angular spin current is different. From the density $\vec{j}_{\omega}(\mathbf{r}, t)$, it is meaningless to determine how much the angular spin current flowing through a surface $S$, because the angular spin current describes the rotational motion not the movement. On the other hand, one can calculate the total angular spin current $\vec{I}_{\omega}^{V}$ in a volume $V$ from $\vec{j}_{\omega}: \vec{I}_{\omega}^{V}=\iiint_{V} \vec{j}_{\omega}(\mathbf{r}, t) d V$. (4) If the system is in a steady state, $\mathbf{j}_{s}$ and $\vec{j}_{\omega}$ are independent of the time $t$, and $\frac{d}{d t} \vec{s}(\mathbf{r}, t)=0$. Then the spin continuity equation (11) reduces to: $\nabla \bullet \mathbf{j}_{s}=\vec{j}_{\omega}$ or $\oint_{S} d \vec{S} \bullet \mathbf{j}_{s}=\iiint_{V} \vec{j}_{\omega} d V$. This means that the total linear spin current flowing out of a closed surface is equal to the total angular spin current enclosed. If to further consider a quasi one dimensional (1D) system (see Fig.1e), then one has $\vec{I}_{s}^{S^{\prime}}-\vec{I}_{s}^{S}=\vec{I}_{\omega}^{V}$. (5) It is easy to prove that the spin currents in the present definitions of Eqs. $(13,14)$ are invariant under a space coordinate transformation. (6) The linear spin current density $\mathbf{j}_{s}=\operatorname{Re}\left\{\Psi^{\dagger} \vec{v} \hat{\vec{s}} \Psi\right\}$ gives both the spin direction and the direction of spin movement, so it completely describes the translational motion. However, the angular spin current density, $\vec{j}_{\omega}=\operatorname{Re}\left\{\Psi^{\dagger} \frac{d \hat{\vec{s}}}{d t} \Psi\right\}=\operatorname{Re}\left\{\Psi^{\dagger} \vec{\omega} \times \hat{\vec{s}} \Psi\right\}$ involves the vector product of $\vec{\omega} \times \hat{\vec{s}}$, not the tensor $\vec{\omega} \hat{\vec{s}}$. Is it correct or sufficient to describe the rotational motion? For example, the rotational motion of Fig.1c with the spin $\vec{s}$ in the $y$ direction and the angular velocity $\vec{\omega}$ in the $-z$ direction is different from the one in Fig.1d in which $\vec{s}$ is in the $z$ direction and $\vec{\omega}$ is in the $y$ direction, but their angular spin currents are completely the same. Shall we distinguish them? It turns out that the physical results produced by the above two rotational motions (Fig.1c and 1d) are indeed the same. For instance, the induced electric field by them is identical since a spin $\vec{s}$ has only the direction but no size (see detail discussion below). Thus, the vector $\vec{j}_{\omega}$ is sufficient to describe the rotational motion, and no tensor is necessary.

Recently, theoretic studies have suggested that the (linear) spin current can induce an electric field $\vec{E}[15,16]$. Can the angular spin current also induce an electric field? If so this gives a way of detecting the angular spin current. Following, we study this question by using the method of equivalent magnetic charge [17]. Let us consider a steadystate angular spin current element $\vec{j}_{\omega} d V$ at the origin. Associated with the spin $\vec{s}$, there is a magnetic moment (MM) $\vec{m}=g \mu_{B} \vec{\sigma}=\frac{2 g \mu_{B}}{\hbar} \vec{s}$ where $\mu_{B}$ is the Bohr magneton. Thus, corresponding to $\vec{j}_{\omega}$, there is also a angular MM current $\vec{j}_{m \omega} d V=\frac{2 g \mu_{B}}{\hbar} \vec{j}_{\omega} d V$. From above discussions, we already know that $\vec{j}_{m \omega}$ (or $\vec{j}_{\omega}$ ) comes from the rotational motion of a MM $\vec{m}$ (or $\vec{s}$ ) (see Fig.1c and 1d), and $\vec{j}_{m \omega}=\vec{\omega} \times \vec{m}$ (or $\vec{j}_{\omega}=\vec{\omega} \times \vec{s}$ ). Under the method of equivalent magnetic charge, the MM $\vec{m}$ is equivalent to two magnetic charges: one with magnetic charge $+q$ located at $\delta \hat{n}_{m}$ and the other with $-q$ at $-\delta \hat{n}_{m}$ (see Fig.1f). $\hat{n}_{m}$ is the unit vector of $\vec{m}$ and $\delta$ is a tiny length. The angular MM current $\vec{j}_{m \omega}$ is equivalent to two magnetic charge currents: one is $\vec{j}_{+q}=\hat{n}_{j} q \delta|\vec{\omega}| \sin \theta$ at the location $\delta \hat{n}_{m}$, the other is $\vec{j}_{-q}=\hat{n}_{j} q \delta|\vec{\omega}| \sin \theta$ at $-\delta \hat{n}_{m}$ (see Fig.1f), with $\hat{n}_{j}$ being the unit vector of $\vec{j}_{m \omega}$ and $\theta$ the angle between $\vec{\omega}$ and $\vec{m}$. In our previous work, 16 we have given the formulae of the electric field induced by a magnetic charge current. The electric field induced by $\vec{j}_{m \omega} d V$ can be calculated by adding the contributions from the two magnetic charge currents. Let $\delta \rightarrow 0$, and note that $2 q \delta \rightarrow|\vec{m}|$ and $|\vec{\omega}||\vec{m}| \sin \theta=\left|\vec{j}_{m \omega}\right|$, we obtain the electric field $\vec{E}_{\omega}$ generated by an element of the angular spin current $\vec{j}_{\omega} d V$ :

$$
\vec{E}_{\omega}=\frac{-\mu_{0}}{4 \pi} \int \frac{\vec{j}_{m \omega} d V \times \mathbf{r}}{r^{3}}=\frac{-\mu_{0} g \mu_{B}}{h} \int \vec{j}_{\omega} d V \times \frac{\mathbf{r}}{r^{3}}
$$

We also rewrite the electric field $\vec{E}_{s}$ generated by an element of the linear spin current using the tensor $\mathbf{j}_{s}: 16$

$$
\vec{E}_{s}=\frac{-\mu_{0} g \mu_{B}}{h} \nabla \times \int \mathbf{j}_{s} d V \bullet \frac{\mathbf{r}}{r^{3}},
$$


Below we emphasize three points: (i) In the large $r$ case, the electric field $\vec{E}_{\omega}$ decays as $1 / r^{2}$. Note that the field from a linear spin current $\vec{E}_{s}$ goes as $1 / r^{3}$. In fact, in terms of generating an electric field, the angular spin current is as effective as a magnetic charge current. (ii) In the steady-state case, the total electric field $\vec{E}_{T}=\vec{E}_{\omega}+\vec{E}_{s}$ contains the property: $\oint_{C} \vec{E}_{T} \bullet d \vec{l}=0$, where $C$ is an arbitrary close contour not passing through the region of spin current. However, for each $\vec{E}_{\omega}$ or $\vec{E}_{s}, \oint_{C} \vec{E}_{\omega} \bullet d \vec{l}$ or $\oint_{C} \vec{E}_{s} \bullet d \vec{l}$ can be non-zero. (iii) As mentioned above, a angular spin current $\vec{j}_{\omega}$ may consist of different $\vec{\omega}$ and $\vec{s}$ (see Fig.1c and 1d). However, the resulting electric field only depends on $\vec{j}_{\omega}=\vec{\omega} \times \vec{s}$. This is because a spin vector contains only a direction and a magnitude, but not a spatial size (i.e. the distance $\delta$ approaches to zero). In the limit $\delta \rightarrow 0$, both magnetic charge currents $\overrightarrow{j_{ \pm q}}$ reduce to $\vec{\omega} \times \vec{m} / 2$ at the origin. Therefore, the overall effect of the rotational motion is only related to $\vec{\omega} \times \vec{m}$, not separately on $\vec{\omega}$ and $\vec{m}$. Hence it is enough to describe the spin rotational motion by using a vector $\vec{\omega} \times \vec{s}$, instead of a tensor $\vec{\omega} \vec{s}$.

Now we give an example of applying the above formulas, Eqs.(13,14), to calculate the spin currents and the electric fields generated by them. Let us consider a quasi 1D quantum wire having the Rashba spin orbit coupling, and its Hamiltonian is:

$$
H=\frac{\vec{p}^{2}}{2 m}+V(y, z)+\frac{\alpha}{\hbar} \sigma_{z} p_{x},
$$

Here the other Rashba term $-\frac{\alpha}{\hbar} \sigma_{x} p_{z}$ is neglected because z-direction is quantized [6]. Let $\Psi$ be a stationary wave function

$$
\Psi(\mathbf{r})=\frac{\sqrt{2}}{2}\left(\begin{array}{c}
e^{i\left(k-k_{R}\right) x} \\
e^{i\left(k+k_{R}\right) x}
\end{array}\right) \varphi(y, z),
$$

where $k_{R} \equiv \alpha m / \hbar^{2}$ and $\varphi(y, z)$ is the bound state wave function in the confined $y$ and $z$ directions. $\Psi(\mathbf{r})$ represents the spin precession in the $x$ - $y$ plane while moving along the $x$ axis and this wave function was discussed in recent studies. 6, 7] Using Eqs.(13,14), the spin current densities of the wave function $\Psi(\mathbf{r})$ are easily obtained. There are only two non-zero elements of $\mathbf{j}_{s}(\mathbf{r})$ :

$$
\begin{aligned}
& j_{s x x}(\mathbf{r})=\left(\hbar^{2} k / 2 m\right)|\Psi(y, z)|^{2} \cos 2 k_{R} x, \\
& j_{s x y}(\mathbf{r})=\left(\hbar^{2} k / 2 m\right)|\Psi(y, z)|^{2} \sin 2 k_{R} x .
\end{aligned}
$$

The non-zero elements of $\vec{j}_{\omega}(\mathbf{r})$ are:

$$
\begin{aligned}
& j_{\omega x}(\mathbf{r})=-\left(\hbar^{2} k k_{R} / m\right)|\Psi(y, z)|^{2} \sin 2 k_{R} x \\
& j_{\omega y}(\mathbf{r})=\left(\hbar^{2} k k_{R} / m\right)|\Psi(y, z)|^{2} \cos 2 k_{R} x
\end{aligned}
$$

Those spin current densities confirm with the intuitive picture of an electron motion, precession in the $x-y$ plane and movement in the $x$ direction (see Fig.2a). Moreover, they indeed satisfy $-\nabla \bullet \mathbf{j}_{s}(\mathbf{r})+\vec{j}_{\omega}(\mathbf{r})=0$.

Let us calculate the induced electric fields at the location $\mathbf{r}=(x, y, z)$ by the above spin currents. Substituting the spin currents of Eqs.(19-22) into Eqs. $(15,16)$ and assuming the transverse sizes of the $1 \mathrm{D}$ wire are much smaller than $\sqrt{y^{2}+z^{2}}$, the induced fields $\vec{E}_{\omega}$ and $\vec{E}_{s}$ can be obtained as:

$$
\begin{aligned}
& \vec{E}_{\omega}=-a K_{1}(\beta) \sin \tilde{x}\left(\frac{\tilde{z} \cos \tilde{x}}{\beta \sin \tilde{x}}, \frac{\tilde{z}}{\beta},-\frac{\tilde{y}}{\beta}-\frac{K_{0}(\beta)}{K_{1}(\beta)}\right), \\
& \vec{E}_{s}=a \frac{K_{1}}{\beta} \sin \tilde{x}\left(0, \frac{2 \tilde{z} \tilde{y}}{\beta^{2}}+\tilde{z} F(\beta), \frac{\tilde{z}^{2}-\tilde{y}^{2}}{\beta^{2}}-\tilde{y} F(\beta)\right),
\end{aligned}
$$


where $\tilde{i}=2 k_{R} i(i=x, y, z), \beta=\sqrt{\tilde{y}^{2}+\tilde{z}^{2}}, F(\beta)=1+\frac{\tilde{y} K_{0}(\beta)}{\beta K_{1}(\beta)}$, the constant $a=2 \mu_{0} g \mu_{B} \hbar k k_{R}^{2} \rho_{s} / \pi m, \rho_{s}$ is the linear density of moving electrons under the bias of an external voltage, and $K_{0}$ and $K_{1}$ are the Bessel functions. The total electric field is: $\vec{E}_{T}=\vec{E}_{\omega}+\vec{E}_{s}=-\frac{a}{2 k_{R}} \nabla\left\{\frac{\tilde{z} \sin \tilde{x}}{\beta} K_{1}(\beta)\right\}$. The total electric field $\vec{E}_{T}$ represents the one generated by a 1D wire of electric dipole moment $\vec{p}_{e}=(0,0, c \sin \tilde{x})$ at the $x$ axis (see Fig.2b), where $c$ is a constant. It is obvious that $\nabla \times \vec{E}_{T}=0$, i.e. $\oint_{C} \vec{E}_{T} \bullet d \vec{l}=0$. However, in general $\oint_{C} \vec{E}_{\omega} \bullet d \vec{l}$ and $\oint_{C} \vec{E}_{s} \bullet d \vec{l}$ are separately non-zero. Finally, we estimate the magnitude of $\vec{E}_{T}$. We use parameters consistent with realistic experimental samples. Take the Rashba parameter $\alpha=3 \times 10^{-11} \mathrm{eVm}$ (corresponding to $k_{R}=1 / 100 \mathrm{~nm}$ for $m=0.036 m_{e}$ ), $\rho_{s}=10^{6} / \mathrm{m}$ (i.e. one moving electron per $1000 \mathrm{~nm}$ in length), and $k=k_{F}=10^{8} / \mathrm{m}$. The electric potential difference between the two points A and B (see Fig.2b) is about $0.01 \mu V$, where the positions of A and B are $\frac{1}{2 k_{R}}\left(\frac{\pi}{2}, 0,0.01\right)$ and $\frac{1}{2 k_{R}}\left(\frac{3 \pi}{2}, 0,0.01\right)$. This value of the potential is measurable with today's technology. Furthermore, with the above parameters the electric field $\vec{E}_{T}$ at $\mathrm{A}$ or $\mathrm{B}$ is about $10 \mathrm{~V} / \mathrm{m}$ which is rather large.

In summary, we have introduced the angular spin current and its role in the spin continuity equation. We point out that the angular spin current can also induce an electric field and its $\vec{E}$ field scales as $1 / r^{2}$ at large $r$.

Acknowledgments: We gratefully acknowledge financial support from the Chinese Academy of Sciences and NSFC under Grant No. 90303016 and No. 10474125. XCX is supported by US-DOE under Grant No. DE-FG02-04ER46124 and NSF-MRSEC under DMR-0080054.

[*] lectronic address: sunqf@aphy.iphy.ac.cn

[1] S.A. Wolf, et al., Science 294, 1488 (2001); G.A. Prinz, Science 282, 1660 (1998).

[2] I. Zutic, J. Fabian, and S. Das Sarma, Rev. Mod. Phys. 76, 323 (2004).

[3] S. Murakami, N. Nagaosa, S.-C. Zhang, Science 301, 1348 (2003); Phys. Rev. B 69, 235206 (2004); Z.F. Jiang, et al., cond-mat/0410420 (2004).

[4] J. Sinova, et al., Phys. Rev. Lett. 92, 126603 (2004); B.K. Nikolic, et al. cond-mat/0412595 (2004).

[5] S.-Q. Shen, et al., Phys. Rev. Lett. 92, 256603 (2004); cond-mat/0410169 (2004); S. Murakami, et al., Phys. Rev. Lett. 93, 156804 (2004); S.-P. Kou, X.-L. Qi, and Z.-Y. Weng, cond-mat/0412146 (2004).

[6] S. Datta and B. Das, Appl. Phys. Lett. 56, 665 (1990).

[7] T. Matsuyama, et al., Phys. Rev. B 65, 155322 (2002); F. Mireles and G. Kirczenow, ibid, 64, 024426 (2001).

[8] D. Culcer, et al. Phys. Rev. Lett. 93, 046602 (2004).

[9] S. Zhang and Z. Yang, Phys. Rev. Lett. 94066602 (2005).

[10] Notice that those results, Eqs.(11, 13, and 14), have the generality, and they are not dependent on the specific Hamiltonian (4). For example, in the case of the existence of the vector potential $\vec{A}$, the general spin-orbit $\operatorname{coupling} \alpha \hat{\vec{\sigma}} \bullet[\vec{p} \times \nabla V(\mathbf{r})]$, and so on, the results all hold.

[11] E.I. Rashba, Fiz. Tverd. Tela (Leningrad) 2, 1224 (1960) [Solid State Ionics 2, 1109 (1960)].

[12] Y.A. Bychkov and E.I. Rashba, J. Phys. C 17, 6039 (1984).

[13] $\vec{j}_{\omega}$ is called the spin torque in the work $[8]$ in which the semi-classical approach to the spin continuity equation is discussed. Here we consider that $\vec{j}_{\omega}$ describes the rotational motion of a spin, thus named the angular spin current.

[14] From $\mathbf{j}_{s}(\mathbf{r}, t)$, the total translation spin current along $i$ direction $(i=x, y, z)$ is: $\vec{I}_{s i}(i, t)=\iint d S i \hat{i} \mathbf{j}_{s}(\mathbf{r}, t)$. To assume $\vec{I}_{s i}(i, t)$ independent on $t$ and $i$ (e.g. in the case of the steady state and without spin flip), one has: $\vec{I}_{s i}=\frac{1}{L} \iiint d V \hat{i} \bullet \mathbf{j}_{s}(\mathbf{r}, t)=$ $\frac{1}{L} \iiint d V \operatorname{Re} \Psi^{\dagger} \hat{v}_{i} \hat{\vec{s}} \Psi=\frac{1}{L} \iiint d V \Psi^{\dagger} \frac{1}{2}\left(\hat{v}_{i} \hat{\vec{s}}+\hat{\vec{s}}_{i}\right) \Psi=<\frac{1}{2}\left(\hat{v}_{i} \hat{\vec{s}}+\hat{\vec{s}}_{i}\right)>$, where $L$ is sample length in $i$-direction. This definition is the same as in recent publications [4].

[15] J.E. Hirsch, Phys. Rev. B 42, 4774 (1990); 60, 14787 (1999); F. Meier and D. Loss, Phys. Rev. Lett. 90, 167204 (2003); F. Schutz, M. Kollar, and P. Kopietz, ibid. 91, 017205 (2003).

[16] Q.-f. Sun, H. Guo, and J. Wang, Phys. Rev. B 69, 054409 (2004).

[17] For example, see Introduction to Electrodynamics (Prentice-Hall, Englewood Cliffs, NJ, 1989). 
FIG. 1: (Color online) (a) Schematic diagram for a classic vector flow. (b) The linear spin current element $j_{s, x y}$. (c) and (d) The angular spin current element $j_{\omega, x}$. (e) The spin current in a quasi $1 \mathrm{D}$ quantum wire. (f) The currents of two magnetic charges that are equivalent to a angular MM current.

FIG. 2: (Color online) (a) Schematic diagram for the spin precession in the $x$ - $y$ plane while moving along the $x$ axis. (b) A 1D wire of electric dipole moment $\vec{p}_{e}$. This configuration will generate an electric field equivalent to the field from the spin currents in (a). 

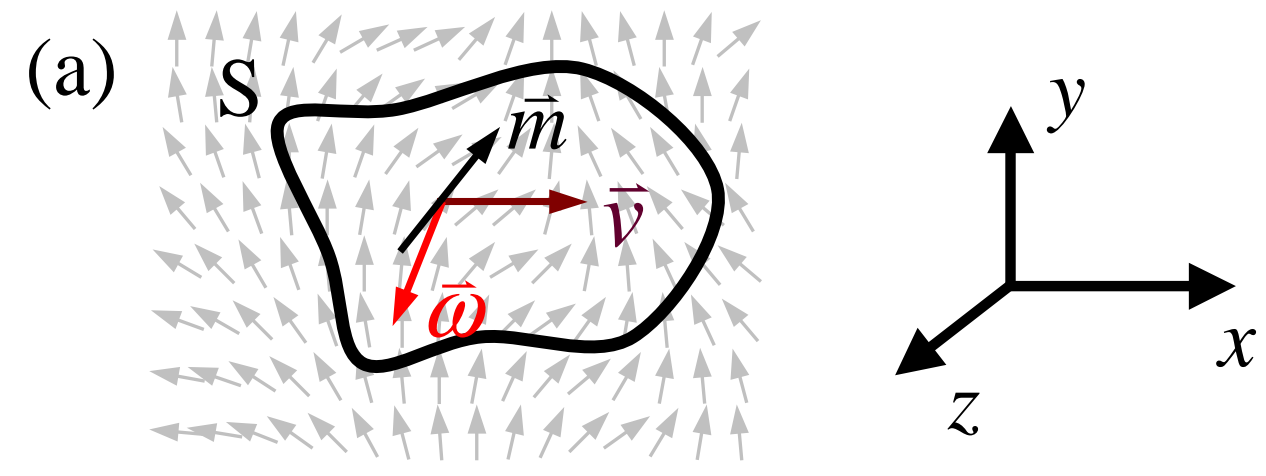

(b)

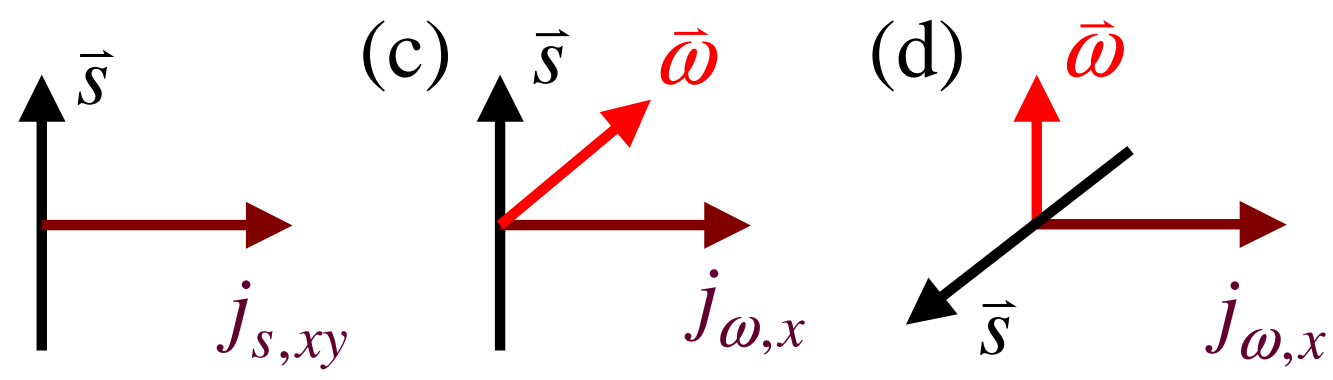

(e)

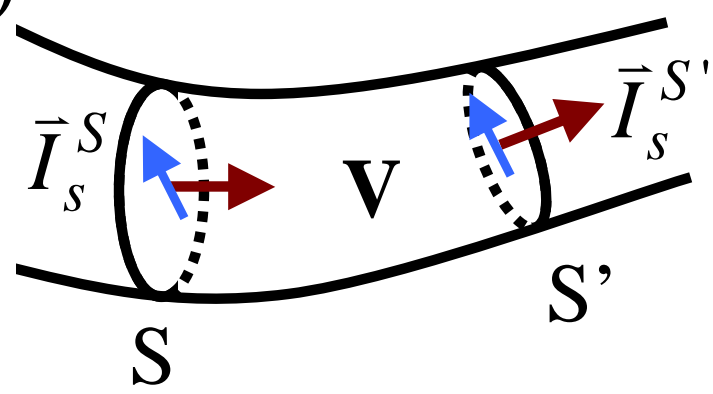

(f)

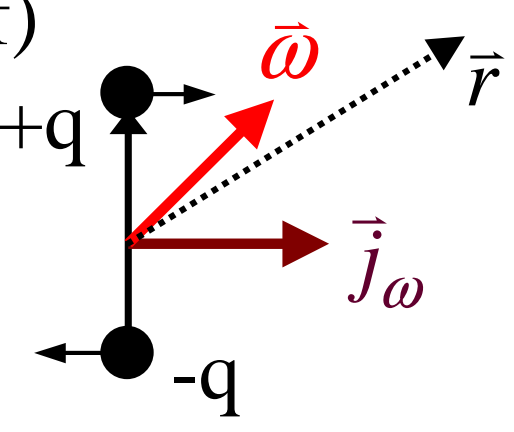



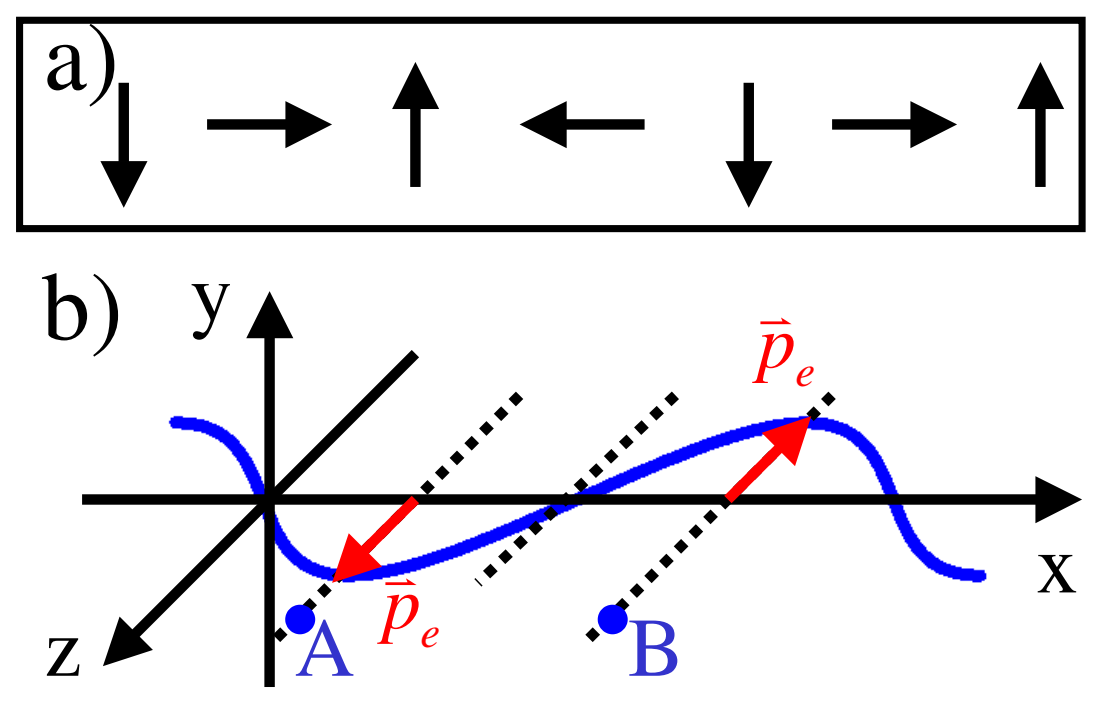